\title{
The Correlation Characteristics of Polarization Backscattering Matrix of Dense Chaff Clouds
}

\author{
Bo TANG \\ School of Computer and Communication Engineering, University of Science and Technology Beijing, Xueyuanlu 30, \\ Beijing, China \\ tangbo@ustb.edu.cn
}

Submitted April 10, 2017 / Accepted October 26, 2017

\begin{abstract}
This paper studied the correlation characteristics of the polarization backscattering matrix of the dense chaff cloud with uniform orientation and location distributions in a circular symmetry region. Based on the theoretical analysis and numerical experiments, the correlation coefficients of the four elements in the polarization backscattering matrix are obtained, and the results indicate that the cross to co-polar correlation coefficient is still zero; and that the sum of the co-polar cross-correlation coefficient and the two times of linear depolarization ratio equals one. The results are beneficial for better understanding of the backscattering characteristics of dense chaff clouds, and are useful in the application of jamming recognition in radar electronic warfare. Numerical experiments are performed by using the method of moments.
\end{abstract}

\section{Keywords}

Dense chaff clouds, polarization characteristics, electronic warfare, radar

\section{Introduction}

As an effective passive countermeasure, chaff (or dipoles) clouds have been playing an important role in electronic warfare [1], scattering communications [2], [3] and other applications, for example, being used as composite materials [4]. Chaff usually has a simple structure of a metal wire. Chaff clouds, a number of dipoles distributed in the radar observation space, are initially assumed as sparse, that is, the average spacing between dipoles is more than two working wavelengths [5]. Radar cross sections (RCS) for sparse chaff clouds were studied in [6], [7]. With the increasing ability of loading power of transport vehicles, dense chaff clouds are often encountered in practices for better jamming effect. Research on dense chaff clouds has received great concern for recent years. A method of equivalent conductor (EC) is presented to calculate RCS in paper [8] by considering the dense chaff cloud as a homogeneous material. Through the boundary condition, the equivalent electric parameter is obtained for a dense chaff cloud. A generalized EC method for arbitrary orientation of chaff is presented in paper [9] as an extent of EC method.
Besides these, the polarization characteristics of chaff clouds are much more focused on for their essentials in chaff jamming recognition and suppression. The polarization characteristics of single dipole and sparse chaff clouds have been studied thoroughly, such as the correlation characteristics of the polarized backscattered field [10] and the polarization characteristics of echoes [11]; the correlation of the received voltage with different polarization [12]; the correlation of received field with different polarization [13]; the polarization characteristics for Gaussian oriented chaff clouds [14].

Polarization characteristics play an important role in the jamming recognition and suppression in radar electronic warfare [15-18]. The scattering characteristics of the dense chaff clouds are not always the same as that of the sparse chaff clouds. For example, the RCS of the dense chaff clouds is not the sum of the dipoles' RCS due to the coupling among the dipoles [5]. The conclusions of sparse clouds cannot be directly extended to the case of dense clouds. The study of the polarization characteristics of the dense chaff clouds is necessary for the chaff jamming recognition and suppression.

This paper focuses on the polarization correlation characteristics of dense chaff clouds with uniform orientation and location distributions in circular symmetry region. Through investigation in correlation characteristics of the polarization backscattering matrix of dense chaff clouds, meaningful conclusions are drown. The results of numerical computation using the method of moments (MoM) [19] and Pocklington integral equation [20] are given, and the numerical results are in agreement with the analytical conclusions given in this paper.

\section{The Sparse Chaff Clouds}

In the coordinates system shown in Fig. 1, the polarization backscattering matrix can be expressed as [21]

$$
\boldsymbol{S}=\left[\begin{array}{ll}
S_{11} & s_{12} \\
S_{21} & s_{22}
\end{array}\right]
$$

Based on the reciprocity principle, $s_{12}=s_{21}$ holds [21]. 


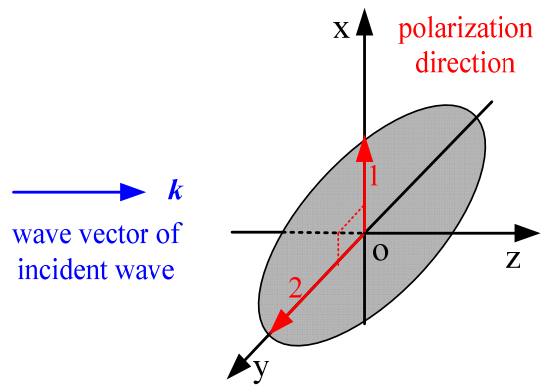

Fig. 1. Coordinates system (Origin is the center of chaff clouds).

Since $s_{12}=s_{21}$, let $S^{\prime}=\left[s_{11}, s_{12}, s_{22}\right]$. The covariance matrix of $\boldsymbol{S}^{\prime}$ is

$$
\boldsymbol{C}=\left[\begin{array}{lll}
\left\langle s_{11} s_{11}^{*}\right\rangle & \left\langle s_{11} s_{12}^{*}\right\rangle & \left\langle s_{11} s_{22}^{*}\right\rangle \\
\left\langle s_{12} s_{11}^{*}\right\rangle & \left\langle s_{12} s_{12}^{*}\right\rangle & \left\langle s_{12} s_{22}^{*}\right\rangle \\
\left\langle s_{22} s_{11}^{*}\right\rangle & \left\langle s_{22} s_{12}^{*}\right\rangle & \left\langle s_{22} s_{22}^{*}\right\rangle
\end{array}\right]
$$

where $\langle\cdot\rangle$ represent the ensemble average.

For the single chaff or sparse chaff clouds with uniform orientation distribution, $\boldsymbol{C}$ can be obtained that [10], [22], [23]

$$
C=\left\langle s_{11} s_{11}^{*}\right\rangle\left[\begin{array}{ccc}
1 & 0 & 1 / 3 \\
0 & 1 / 3 & 0 \\
1 / 3 & 0 & 1
\end{array}\right] .
$$

The experiments of a variety of distributed targets (bare soil surface, vegetation-covered terrain, etc.) show that $s_{11}$ is independent of $s_{12}$ [24]. As seen in (3), this conclusion holds for the sparse chaff cloud [10]. So, the following is to determine whether this conclusion holds for dense chaff clouds.

\section{The Analysis for Dense Chaff Clouds Based on MOM}

The coupling among dipoles cannot be ignored for dense chaff clouds. The backscattered fields of dense chaff clouds are computed by MoM. In the numerical computation, the dense chaff cloud with $M$ dipoles is modeled as a whole object, and every dipole is divided into $N$ segments linearly. $N$ is an odd number. $Z_{i m, h n}$ is the mutual impendence between the $m$ th segment in the $i$ th dipole and the $n$th segment in the $h$ th dipole. $i, h=1, \ldots, M$, and $-(N-1) / 2 \leq m \leq(N-1) / 2,-(N-1) / 2 \leq n \leq(N-1) / 2$. The electric current on the $m$ th segment in the $i$ th dipole can generate the electric field on the $n$th segment in the $h$ th dipole.

The orientation unit vector of $i$ th dipole is $\boldsymbol{e}_{i}$, and so does for $\boldsymbol{e}_{h}$, as seen in Fig. 2 .

$$
\begin{array}{r}
\boldsymbol{e}_{i}=\boldsymbol{e}_{\mathrm{x}} \sin \theta_{i} \cos \varphi_{i}+\boldsymbol{e}_{\mathrm{y}} \sin \theta_{i} \sin \varphi_{i}+\boldsymbol{e}_{\mathrm{z}} \cos \theta_{i}, \\
\boldsymbol{e}_{h}=\boldsymbol{e}_{\mathrm{x}} \sin \theta_{h} \cos \varphi_{h}+\boldsymbol{e}_{\mathrm{y}} \sin \theta_{h} \sin \varphi_{h}+\boldsymbol{e}_{\mathrm{z}} \cos \theta_{h}
\end{array}
$$

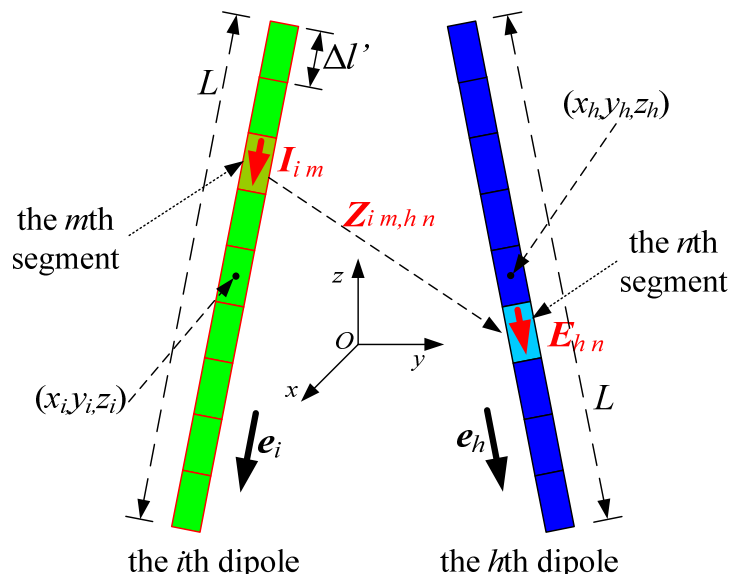

Fig. 2. Segments and orientations of dipoles.

where $\boldsymbol{e}_{\mathrm{x}}, \boldsymbol{e}_{\mathrm{y}}$ and $\boldsymbol{e}_{\mathrm{z}}$ are coordinates unit vectors in xyz coordinates system. $\theta_{i}$ and $\varphi_{i}$ are the angle coordinates variables in the spherical coordinate system. The center of the $i$ th dipole is located on $\left(x_{i}, y_{i}, z_{i}\right)$, the center of the $h$ th dipole is located on $\left(x_{h}, y_{h}, z_{h}\right)$.

Based on Pocklington integral equation [20], it is obtained that

$$
\begin{aligned}
& Z_{i m, h n}(\boldsymbol{r})= \\
& \boldsymbol{e}_{\boldsymbol{h}} \cdot\left[-\frac{1}{\mathrm{j} \omega \varepsilon_{0}}\left(\nabla \nabla \cdot+k^{2}\right) \frac{\exp \left(-\mathrm{j} k\left|\boldsymbol{r}-\boldsymbol{r}^{\prime}\right|\right)}{4 \pi\left|\boldsymbol{r}-\boldsymbol{r}^{\prime}\right|} \boldsymbol{e}_{\boldsymbol{i}} I^{\prime}\left(\boldsymbol{r}^{\prime}\right) \Delta l^{\prime}\right]
\end{aligned}
$$

where

$$
\begin{aligned}
\left|\boldsymbol{r}-\boldsymbol{r}^{\prime}\right|= & \begin{array}{r}
\left(x_{i}+l_{m} \sin \theta_{i} \cos \varphi_{i}-x_{h}-l_{n} \sin \theta_{h} \cos \varphi_{h}\right)^{2} \\
+\left(y_{i}+l_{m} \sin \theta_{i} \sin \varphi_{i}-y_{h}-l_{n} \sin \theta_{h} \sin \varphi_{h}\right)^{2} \\
+\left(z_{i}+l_{m} \cos \theta_{i}-z_{h}-l_{n} \cos \theta_{h}\right)^{2}
\end{array} \\
\boldsymbol{r}= & \boldsymbol{e}_{\boldsymbol{x}}\left(x_{i}+l_{m} \sin \theta_{i} \cos \varphi_{i}\right)+ \\
& \boldsymbol{e}_{\boldsymbol{y}}\left(y_{i}+l_{m} \sin \theta_{i} \sin \varphi_{i}\right)+\boldsymbol{e}_{z}\left(z_{i}+l_{m} \cos \theta_{i}\right), \\
\boldsymbol{r}^{\prime}= & \boldsymbol{e}_{\boldsymbol{x}}\left(x_{h}+l_{n} \sin \theta_{h} \cos \varphi_{h}\right)+ \\
& +\boldsymbol{e}_{\boldsymbol{y}}\left(y_{h}+l_{n} \sin \theta_{h} \sin \varphi_{h}\right)+\boldsymbol{e}_{z}\left(z_{h}+l_{n} \cos \theta_{h}\right)
\end{aligned}
$$

where $\boldsymbol{r}$ is the position vector of the electric field, and $\boldsymbol{r}^{\prime}$ is the position vector of the electric current. $\omega$ is the angular frequency. $k$ is the wave number of the electromagnetic wave. $\nabla$ is a vector differential operator. The current base function used here is a rectangular pulse waveform, that is, $I^{\prime}\left(\boldsymbol{r}^{\prime}\right)=1 . \Delta l^{\prime}$ is the length of the segment. $l_{m}=m L / N$ and $l_{n}=n L / N$, where $L$ is the length of chaff. Denote $p=i N+m, q=h N+n$. The elements of impendence matrix $\boldsymbol{Z}$ can be denoted as $Z_{p, q}=Z_{i N+m, h N+n}=Z_{i m, h n}$. Therefore $Z_{p, q}$ is the function of $x_{i}-x_{h}, y_{i}-y_{h}, z_{i}-z_{h}, \sin \theta_{i} \cos \varphi_{i}$, $\sin \theta_{h} \cos \varphi_{h}, \sin \theta_{i} \sin \varphi_{i}, \sin \theta_{h} \sin \varphi_{h}, \cos \theta_{i}$, and $\cos \theta_{h}$.

Let $\boldsymbol{A}=\boldsymbol{Z}^{-1}$. Then $A_{p, q}=A_{i m, h n}$ is the function of $x_{u}-x_{v}, y_{u}-y_{v}, z_{u}-z_{v}, \sin \theta_{u} \cos \varphi_{u}, \sin \theta_{v} \cos \varphi_{v}, \sin \theta_{u} \sin \varphi_{u}$, $\sin \theta_{v} \sin \varphi_{v}, \cos \theta_{u}$, and $\cos \theta_{v}$, where $u, v=1, \ldots, M$. That is, every element of $\boldsymbol{A}$ is the function of all dipoles' parame- 
ters. According to the vector synthetic and decomposition [19-21], then the coefficient $s_{11}$ can be written as

$$
\begin{aligned}
& s_{11}= \\
& \Gamma \sum_{i=1}^{M} \sum_{h=1}^{M} B_{i, h} \sin \theta_{h} \cos \varphi_{h} \sin \theta_{i} \cos \varphi_{i} \exp \left[-\mathrm{j} k\left(z_{i}+z_{h}\right)\right], \\
& B_{i, h}=\sum_{m=1}^{N} \sum_{n=1}^{N} A_{i m, h n} \exp \left[-\mathrm{j} k\left(l_{m} \cos \theta_{i}+l_{n} \cos \theta_{h}\right)\right]
\end{aligned}
$$

where $\Gamma$ is a factor only related to the distance between radar and the center of the chaff cloud. $\sin \theta_{h} \cos \varphi_{h}$ is the project factor from the polarization direction of incident wave to the dipole orientation. $\sin \theta_{i}$ is the field pattern function of current unit; $\cos \varphi_{i}$ is the project factor of backscattered field on direction $1 ; \exp \left[-\mathrm{j} k\left(z_{i}+\mathrm{z}_{h}\right)\right]$ is due to the time-delay of incident wave and backscattered wave. In the same way, $s_{22}$ and $s_{12}$ can be obtained as

$s_{22}=$

$$
\begin{aligned}
& \Gamma \sum_{i=1}^{M} \sum_{h=1}^{M} B_{i, h} \sin \theta_{h} \sin \varphi_{h} \sin \theta_{i} \sin \varphi_{i} \exp \left[-\mathrm{j} k\left(z_{i}+z_{h}\right)\right], \\
& s_{12}= \\
& \Gamma \sum_{i=1}^{M} \sum_{h=1}^{M} B_{i, h} \sin \theta_{h} \cos \varphi_{h} \sin \theta_{i} \sin \varphi_{i} \exp \left[-\mathrm{j} k\left(z_{i}+z_{h}\right)\right] .
\end{aligned}
$$

For convenience of analysis, dense chaff clouds under the following conditions are concerned here: the chaff orientation and location distributions are uniform, the shape of the chaff cloud is circular symmetrical.

Therefore, $\left\langle s_{11} s_{12}^{*}\right\rangle$ is derived based on the above expressions as

$$
\begin{aligned}
& \left\langle s_{11} s_{12}^{*}\right\rangle= \\
& |\Gamma|^{2} \sum_{i=1}^{M} \sum_{h=1}^{M} \sum_{u=1}^{M} \sum_{v=1}^{M}\left[B_{i, h} B_{u, v}^{*} f(\theta) \cdot \cos \varphi_{h} \cos \varphi_{i} \cos \varphi_{u} \sin \varphi_{v} g(z)\right]
\end{aligned}
$$

where

$$
\begin{gathered}
f(\theta)=\sin \theta_{h} \sin \theta_{i} \sin \theta_{u} \sin \theta_{v}, \\
g(z)=\exp \left[-\mathrm{j} k\left(z_{i}+z_{h}-z_{u}-z_{v}\right)\right] .
\end{gathered}
$$

When the chaff orientation distribution is uniform and the shape of the chaff cloud is circular symmetry, $B_{i, h} B_{u, v}^{*}$ is the even function of the variables in the set $\left(\varphi_{1}, \ldots, \varphi_{M}\right.$, $\left.y_{1}, \ldots, y_{M}\right)$ according to the (6) and (7); and $\cos \varphi_{h} \cos \varphi_{i} \cos \varphi_{u} \sin \varphi_{v}$ is the odd function of variables in the set $\left(\varphi_{1}, \ldots, \varphi_{M}, y_{1}, \ldots, y_{M}\right)$; and $\varphi_{i}(i=1, \ldots, M)$ satisfies the uniform distribution in $[-\pi, \pi]$. Therefore, it is obtained that

$$
\left\langle s_{11} s_{12}^{*}\right\rangle=0 \text {. }
$$

Equation (17) shows that $s_{11}$ is uncorrelated to $s_{12}$, that is, the cross to co-polar correlation coefficient $\left\langle s_{11} s_{12}^{*}\right\rangle / \sqrt{\left\langle s_{11} s_{11}^{*}\right\rangle\left\langle s_{12} s_{12}^{*}\right\rangle}$ is zero. According to the statistical theory, uncorrelation is equal to independence for Gaussian random variables. Because $s_{11}$ and $s_{12}$ satisfy
Gaussian distribution [22], and they are uncorrelated to each other based on the above analysis, therefore they are independent of each other. This conclusion coincides with the experiments of distributed targets mentioned in [24], and it is independent of the length of dipoles.

Based on the circular symmetry of orientation and location, the correlation characteristics should be independent of polarized direction of incident wave. In this circular symmetry situation, it is known that

$$
\left\langle s_{11} s_{11}^{*}\right\rangle=2\left\langle s_{12} s_{12}^{*}\right\rangle+\left\langle s_{11} s_{22}^{*}\right\rangle
$$

which can be seen through a common derivation (see Appendix). Note that $\left\langle s_{11} s^{*}{ }_{11}\right\rangle=\left\langle s_{22} S^{*}{ }_{22}\right\rangle$ due to the circular symmetry. Then, equation (18) means that there is a negative correlation between the co-polar cross-correlation coefficient $\left\langle s_{11} s_{22}^{*}\right\rangle / \sqrt{\left\langle s_{11} s_{11}^{*}\right\rangle\left\langle s_{22} s_{22}^{*}\right\rangle}$ and the linear depolarization ratio $\left\langle\mathrm{s}_{12} s_{12}^{*}\right\rangle /\left\langle s_{11} s_{11}^{*}\right\rangle$.

\section{Numerical Validations}

The polarization backscattering matrix of chaff clouds are computed by MoM and Pocklington integral equation. Taking this approach, a triangular current basis function is used. Every dipole is divided into 37 segments uniformly. Six different cases are computed. Three different shapes of chaff clouds are considered, including sphere, cylinder and cube, and each shape has two different sizes. The number of dipoles in all these cases is the same, which is 200 . The orientation distribution of dipoles is uniform, and the center of each dipole is dispersed uniformly in the cloud. For cylindrical cloud, the wave vector of incident wave is perpendicular to the bottom surface. For cubical cloud, the wave vector of incident wave is perpendicular to one of six surfaces, and the polarized direction of incident wave is parallel to one edge of the surface. 1000 realizations of each case of chaff clouds are computed in order to obtain statistical average value. The results are listed in Tab. 1. The ratios of $\left\langle s_{12} s^{*}\right\rangle$ to $\left\langle s_{11} s_{11}^{*}\right\rangle$ with different number of realizations are shown in Tab. 1. The correlation coefficients of $s_{11}$ and $s_{12}$ (that is, the cross to co-polar correlation coefficient) are shown in Fig. 3. In the table, $\Delta$ is computed by the equation

$$
\Delta=\left[\left\langle s_{11} s_{11}^{*}\right\rangle-2\left\langle s_{12} s_{12}^{*}\right\rangle-\left\langle s_{11} s_{22}^{*}\right\rangle\right] /\left\langle s_{11} s_{11}^{*}\right\rangle .
$$

From Tab. 1, it can be seen that the cross to co-polar correlation coefficients $\left\langle s_{11} s_{12}^{*}\right\rangle / \sqrt{\left\langle s_{11} s_{11}^{*}\right\rangle\left\langle s_{12} s_{12}^{*}\right\rangle}$ approach zeros for all cases. They are not exactly zeros due to the statistical noise. It is in accordance with the theoretical analysis. In Tab. 1, $\Delta$ approach zeros, which means that equation (18) holds. It is in accordance with the theoretical analysis also.

Let $a(n)$ denote the numerical result of $s_{11}$ in the $n$th realization. Let $b(n)$ denote the numerical result of $s_{12}$ in the 


\begin{tabular}{|l|l|l|c|c|c|c|}
\hline Case & Size $(\lambda)$ & Ave. spacing $(\lambda)$ & $\frac{\left\langle s_{11} s_{12}^{*}\right\rangle}{\sqrt{\left\langle s_{11} s_{11}^{*}\right\rangle\left\langle s_{12} s_{12}^{*}\right\rangle}}$ & $\frac{\left\langle s_{12} s_{12}^{*}\right\rangle}{\left\langle s_{11} s_{11}^{*}\right\rangle}$ & $\frac{\left\langle s_{11} s_{22}^{*}\right\rangle}{\sqrt{\left\langle s_{11} s_{11}^{*}\right\rangle\left\langle s_{22} s_{22}^{*}\right\rangle}}$ & $\Delta$ \\
\hline 1 (spherical) & $6 /$ diameter & 0.827 & 0.0529 & 0.2888 & 0.4115 & 0.0109 \\
\hline 2 (cylindrical) & $6,6 /$ diameter, height & 0.947 & 0.0053 & 0.2942 & 0.4163 & -0.0047 \\
\hline 3 (cubical) & 6/ edge length & 1.026 & 0.0148 & 0.2854 & 0.4094 & 0.0198 \\
\hline 4 (spherical) & 8/ diameter & 1.103 & 0.0350 & 0.3152 & 0.3783 & -0.0087 \\
\hline 5 (cylindrical) & $8,8 /$ diameter, height & 1.262 & 0.0274 & 0.3222 & 0.3737 & -0.0172 \\
\hline 6 (cubical) & $8 /$ edge length & 1.368 & 0.0306 & 0.3275 & 0.3428 & -0.0051 \\
\hline
\end{tabular}

Tab. 1. Statistical results.
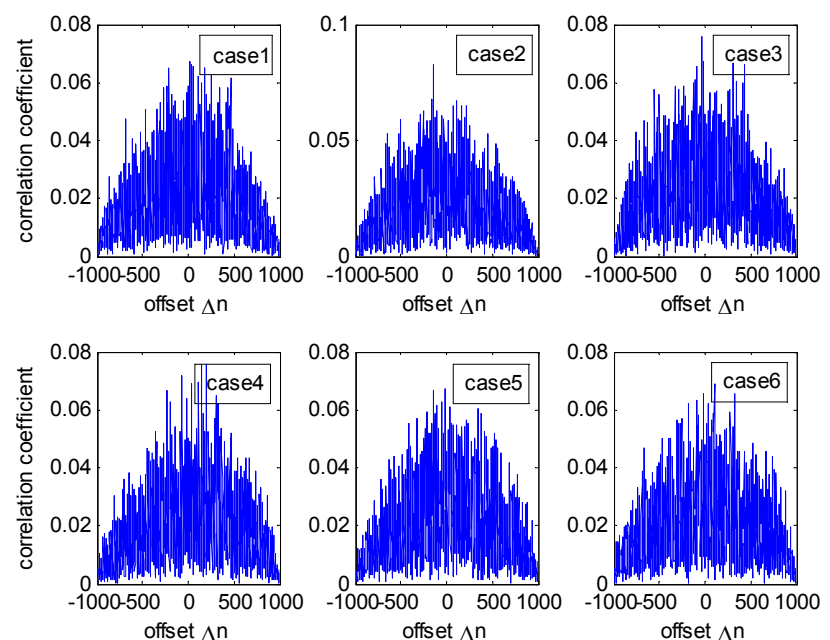

Fig. 3. Correlation coefficients of the computed results of $s_{11}$ and $s_{12}$ (normalized by signal energy).

$n$th realization. The correlation coefficient of $a(n)$ and $b(n)$ is presented as

$$
c(\Delta n)=\frac{\sum_{n=1}^{1000} \operatorname{rect}\left(\frac{n+\Delta n-500}{1000}\right) a(n+\Delta n) b^{*}(n)}{\sqrt{\sum_{n=1}^{1000}\left|a^{2}(n)\right| \sum_{n=1}^{1000}\left|b^{2}(n)\right|}} .
$$

The results of (20) with different $\Delta n$ are shown in Fig. 3. Here, rect() is a rectangle function. $\Delta n$ is the offset, and $-1000 \leq \Delta n \leq 1000$. In Fig. 3, the middle point (where $\Delta n=0$ ) is the computed cross to co-polar correlation coefficients $\left\langle s_{11} s_{12}^{*}\right\rangle / \sqrt{\left\langle s_{11} s_{11}^{*}\right\rangle\left\langle s_{12} s_{12}^{*}\right\rangle}$, the same as shown in Tab. 1. It can be seen that the value of the coefficient $(\Delta n=0)$ is buried by the statistical noise $(\Delta n \neq 0)$ in Fig. 3. So the estimated cross to co-polar correlation coefficient $\left\langle s_{11} s_{12}^{*}\right\rangle / \sqrt{\left\langle s_{11} s_{11}^{*}\right\rangle\left\langle s_{12} s_{12}^{*}\right\rangle}$ can be considered as zero according to the computed data. It is in accordance with the theoretical analysis. Note that $c(\Delta n)$ should be zero theoretically when $\Delta n \neq 0$, because the chaff clouds of different realizations should be independent to each other. So, the computation results of $c(\Delta n)$ for $\Delta n \neq 0$ in Fig. 3 is the statistical noise.

Although it is assumed that the shape of the chaff cloud is circular symmetrical, it is interesting that the result for cube is not obviously different from that for the circular symmetry shape such as sphere and cylinder. So (17) and (18) holds not only for the dense chaff clouds with circular symmetrical shape. That is, $s_{11}$ and $s_{12}$ are independent of each other, and $\left\langle s_{11} s^{*}{ }_{11}\right\rangle$ equals the sum of $2\left\langle s_{12} s_{12}^{*}\right\rangle$ and $\left\langle s_{11} s_{22}^{*}\right\rangle$.

\section{Conclusions}

The correlation characteristics of the polarization backscattering matrix of dense chaff clouds with the uniform orientation and location distributions in circular symmetry region were discussed. It is found that the correlation characteristics of $s_{11}$ and $s_{12}$ for dense chaff clouds are identical to the sparse chaff clouds. According to the theoretical analysis for the dense chaff clouds with the circular symmetry shape, the cross to co-polar correlation coefficient is still zero and that the sum of the co-polar cross-correlation coefficient $\left\langle s_{11} s_{22}^{*}\right\rangle / \sqrt{\left\langle s_{11} s_{11}^{*}\right\rangle\left\langle s_{22} s_{22}^{*}\right\rangle}$ and two times of the linear depolarization ratio $\left\langle s_{12} s_{12}^{*}\right\rangle /\left\langle s_{11} s_{11}^{*}\right\rangle$ equals one. These results are validated by the numerical computation with different shapes of chaff clouds. The results given in this paper are beneficial for better understanding of the backscattering characteristics of dense chaff clouds and are useful in the application of jamming recognition in radar electronic warfare.

\section{Appendix}

It can be assumed that there is an angle $\psi$ between the polarized direction 1 and $\mathrm{x}$ axis in xoy plane generally. (Note that $\psi=0$ in Fig. 1.) Thus, according to the vector synthetic and decomposition, it can be obtained that

$$
\begin{aligned}
& s_{11}= \\
& s_{\mathrm{xx}} \cos ^{2} \psi+s_{\mathrm{xy}} \sin \psi \cos \psi+s_{\mathrm{yx}} \cos \psi \sin \psi+s_{\mathrm{yy}} \sin ^{2} \psi
\end{aligned}
$$

that is, 


$$
s_{11}=s_{\mathrm{xx}} \cos ^{2} \psi+s_{\mathrm{xy}} \sin 2 \psi+s_{\mathrm{yy}} \sin ^{2} \psi
$$

Then

$$
\begin{aligned}
& \left\langle s_{11} s_{11}^{*}\right\rangle= \\
& \left\langle s_{\mathrm{xx}} s_{\mathrm{xx}}^{*}\right\rangle \cos ^{4} \psi+\left\langle s_{\mathrm{yy}} s_{\mathrm{yy}}^{*}\right\rangle \sin ^{4} \psi+\left\langle s_{\mathrm{xy}} s_{\mathrm{xy}}^{*}\right\rangle \sin ^{2} 2 \psi .
\end{aligned}
$$

For the circular symmetry, $\left\langle s_{11} s_{11}^{*}\right\rangle=\left\langle s_{\mathrm{xx}} s_{\mathrm{xx}}^{*}\right\rangle=\left\langle s_{\mathrm{yy}} s_{\mathrm{yy}}^{*}\right\rangle$, $\left\langle s_{11} s_{22}^{*}\right\rangle=\left\langle s_{\mathrm{xx}} s_{\mathrm{yy}}^{*}\right\rangle$ and $\left\langle s_{12} s_{12}^{*}\right\rangle=\left\langle s_{\mathrm{xy}} s_{\mathrm{xy}}^{*}\right\rangle \cdot$ Therefore

$$
\left\langle s_{11} s_{11}^{*}\right\rangle=2\left\langle s_{12} s_{12}^{*}\right\rangle+\left\langle s_{11} s_{22}^{*}\right\rangle \text {. }
$$

Thus, equation (18) is obtained.

\section{Acknowledgments}

This work is supported by the National Natural Science Foundation of China (Grant No. 61471041) and by the Fundamental Research Funds for the Central Universities (FRF-TP-15-028A3).

\section{References}

[1] BUTTERS, B. C. F. Chaff. IEE Proceedings-F - Communications, Radar and Signal Processing, 1982, vol. 129, no. 3, p. 197-201. DOI: $10.1049 /$ ip-f-1:19820030

[2] NEJATINCE, A., VOGT, I. M., GÖSSL, H. Design of chaff and chaff-supported communication systems. IEEE Transactions on Communications, Aug. 1976, vol. 24, no. 8, p.785-803. DOI: 10.1109/TCOM.1976.1093390

[3] HESSEMER, R. A. JR. Scatter communications with radar chaff. IRE Transactions on Antennas and Propagation, 1961, vol. 9, no. 2, p. 211-217. DOI: 10.1109/TAP.1961.1144981

[4] SEO, D.-W., KIM, H.-J., BAE, K.-U., MYUNG, N.-H. The effect of fiber orientation distribution on the effective permittivity of fiber composite materials. Journal of Electromagnetic Waves and Applications, 2010, vol. 24, no. 17-18, p. 2419-2430. DOI: $10.1163 / 156939310793675835$

[5] WICKLIFF, R. G., GARBACZ, R. J. The average backscattering cross section of clouds of randomized resonant dipoles. IEEE Transactions on Antennas and Propagation, 1974, vol. 22, no. 3, p. 503-506. DOI: 10.1109/TAP.1974.1140813

[6] PEEBLES, P. Z. Bistatic radar cross sections of chaff. IEEE Transactions on Aerospace and Electronic Systems, 1984, vol. 20, no. 2, p. 128-140. DOI: 10.1109/TAES.1984.310435

[7] GUO, Y. P., ÜBERALL, H. Bistatic radar scattering by a chaff cloud. IEEE Transactions on Antennas and Propagation, 1992, vol. 40, no. 7, p. 837-841. DOI: 10.1109/8.155750

[8] MARCUS, S. W. Electromagnetic wave propagation through chaff clouds. IEEE Transactions on Antennas and Propagation, 2007, vol. 55, no. 7, p.2032-2042. DOI: 10.1109/TAP.2007.900178

[9] SEO, D.-W., YOO, J.-H., KWON, K. I., et al. Generalized equivalent conductor method for a chaff cloud with an arbitrary orientation distribution. Progress In Electromagnetics Research, 2010, vol. 105, p. 333-346. DOI: 10.2528/PIER10051202

[10] ZRNIC, D. S., RYZHKOV, A. V. Polarimetric properties of chaff. In IEEE International Geoscience and Remote Sensing Symposium IGARSS'03. Toulouse (France), 2003, vol. 4, p. 2359-2361. DOI: 10.1109/IGARSS.2003.1294441
[11] IOANNIDIS, G.A. Model for spectral and polarization characteristics of chaff. IEEE Transactions on Aerospace and Electronic Systems, 1979, vol. 15, no. 5, p. 723-726. DOI: 10.1109/TAES.1979.308862

[12] SHEN, Y., XIE, J. Statistical properties of chaff echoes. Chinese Journal of Radio Science, 1997, vol. 12, no. 1, p. 108-111. (In Chinese)

[13] WANG, X. S., CHEN, Z. J., LI, Y. Z. Polarization scattering characteristics of chaff cloud in outer space. In Proceedings of CIE International Conference on Radar. Bejing (China), 2001, p. 444-448. DOI: 10.1109/ICR.2001.984732

[14] LI, J. L., WANG, X. S., LI, Y. Z. Polarization characteristics of Gaussian oriented chaff clouds. Chinese Journal of Radio Science, 2008, vol. 23, no. 3, p. 389-395. (In Chinese)

[15] TANG, B. LI, H. M., SHENG, X. Q. Jamming recognition method based on the full polarisation scattering matrix of chaff clouds. IET Microwaves, Antennas and Propagation, 2012, vol. 6, no. 13, p. 1451-1460. DOI: 10.1049/iet-map.2012.0297

[16] YANG, Y., XIAO, S. P., FENG, D. J., et al. Polarisation oblique projection for radar seeker tracking in chaff centroid jamming environment without prior knowledge. IET Radar, Sonar and Navigation, 2014, vol. 8, no. 9, p. 1195-1202. DOI: 10.1049/iet-rsn.2013.0388

[17] SHAO, X. H., DU, H., XUE, J. H. A new method of ship and chaff polarization recognition under rain and snow cluster. In 2007 International Workshop on Anti-Counterfeiting, Security and Identification (ASID). Xiamen (China), 2007, p. 142-147. DOI: 10.1109/IWASID.2007.373715

[18] ALKU, L., MOISSEEV, D., AITTOMÄKI, T., et al. Identification and suppression of nonmeteorological echoes using spectral polarimetric processing. IEEE Transactions on Geoscience and Remote Sensing, 2015, vol. 53, no. 7, p. 3628-3638. DOI: 10.1109/TGRS.2014.2380476

[19] HARRINGTON, R. F. Field Computation by Moment Methods. Wiley-IEEE Press, 1993. ISBN: 0780310144

[20] GONG, Z. Modern Electromagnetic Theory. 2nd ed. Peking University Press, 2010. (p. 191-195).

[21] MOTT, H. Remote Sensing with Polarimetric Radar. Wiley-IEEE Press, 2007. (p. 65-68) DOI: 10.1002/0470079819

[22] VAN VLECK, J. H., BLOCH, F., HAMERMESH, M. Theory of radar reflection from wires or thin metallic strips. Journal of Applied Physics, 1947, vol. 18, p. 274-294. DOI: 10.1063/1.1697649

[23] RICHMOND, J., SCHWAB, L., WICKLIFF, R. Tumble-average radar backscatter of some thin-wire chaff elements. IEEE Transactions on Antennas and Propagation, 1974, vol. 22, no. 1, p. 124-126. DOI: 10.1109/TAP.1974.1140734

[24] SARABANDI, K. Derivation of phase statistics from the Mueller matrix. Radio Science, 1992, vol. 27, no. 5, p. 553-560. DOI: 10.1029/92RS00195

\section{About the Author ...}

Bo TANG received the B.S. degree in the Dept. of Physics from Nanjing University (NJU), Nanjing, China, in 2000; and received the Ph.D. degree in the Inst. of Electronics from Chinese Academy of Sciences (CAS), Beijing, China, in 2005. He has been working in the School of Computer and Communication Engineering, University of Science and Technology Beijing (USTB), Beijing, China, since 2005. His research interests include digital communications, microwave imaging, and radio frequency simulation. 